\title{
Electromagnetic Dynamic Response of HL-2M Vacuum Vessel under Plasma Disruption Considering the Electromagneto-Mechanical Coupling Effect
}

\author{
Hongwei Yuan ${ }^{\mathrm{a}}$, Zhensheng Yuan ${ }^{\mathrm{a}}$, Weixin $\mathrm{Li}^{\mathrm{a}}$, Cuixiang Pei ${ }^{\mathrm{a}}$, Shejuan Xie ${ }^{\mathrm{a}}$, Zhenmao Chen ${ }^{\mathrm{a}^{*}}$, \\ Yudong Pan ${ }^{\mathrm{b}}$, Lijun $\mathrm{Cai}^{\mathrm{b}}$ and Jiaxian $\mathrm{Li}^{\mathrm{b}}$ \\ ${ }^{a}$ State Key Laboratory for Strength and Vibration of Mechanical Structures, \\ Shaanxi Engineering Research Center for NDT and Structural Integrity Evaluation \\ Xi'an Jiaotong University, Xi'an 710049, China \\ ${ }^{b}$ Southwestern Institute of Physics, Chengdu 610041, China
}

\begin{abstract}
During plasma disruptions (PDs), transient eddy currents are induced in the HL-2M vacuum vessel (VV) which is a D-shaped, double thin-wall structure. Under the circumstance of high magnetic field, the resulting electromagnetic (EM) forces during PDs are large and the dynamic response of related structures may be violent. In this complicated EM circumstance, the EM-mechanical coupling effect may also have a great influence on the dynamic response of VV structure. In this paper, the EM field and structural dynamic response of HL-2M VV during PDs are simulated by adopting a numerical code of the Lagrangian approach. The Lagrangian approach is on the basis of the Maxwell equations in the Lagrangian description, which treats the coupling behavior of magnetic damping effect without explicitly using the velocity term. This approach can be easily applied to actual structures by updating FEM meshes and reforming coefficient matrices before calculating EM field at each time step. In this work, the disruption plasma currents of operating conditions are simulated by using the DINA code and then the dynamic responses of displacements and stresses of the VV of HL-2M are obtained for both cases with and without considering the coupling effect. The numerical results show that stresses under the disruptions (MD and VDE) are not significant and the coupling effect does not significantly affect the peak dynamic response for the HL-2M problem.
\end{abstract}

Keywords: Vacuum vessel, Coupling effect, Lagrangian approach, Plasma disruption, DINA, Dynamic response

\section{Introduction}

HL-2M, which can realize relative complicated divertor plasma configuration, is an experimental Tokamak device designed by the Southwestern Institute of Physics (SWIP) of China. The major design parameters of HL-2M are 2.5 MA plasma current, $2.2 \mathrm{~T}$ toroidal magnetic field, $1.78 \mathrm{~m}$ major radius, $0.65 \mathrm{~m}$ minor radius, $14 \mathrm{Vs}$ flux swing, 1.8-2.0 elongation, bigger than 0.5 triangularity and $5 \mathrm{~s}$ plasma duration time [1-3]. During the design and construction periods, much attention has been devoted to the electromagnetic (EM) force problem to ensure its structural integrity, especially at the situation of disruption.

During plasma disruptions (PDs), strong transient eddy currents can be induced in the first wall and the vacuum vessel (VV). Interacted with the high confinement magnetic field, large EM forces may be generated and the dynamic responses of related in-vessel structures may be violent. In such a complex EM field environment, the EM-mechanical coupling effect including the magnetic damping and the magnetic stiffness effects may have significant influence on the structural dynamic response. Many works have been done for the design of ITER structural components or other new Tokamak devices by using commercial software such as the ANSYS, ABAQUS etc. However, these softwares cannot properly treat the EM-mechanical coupling effect in a direct way. Since the Team problem 16 was put forward, several studies were conducted on the EM-mechanical coupling analysis [4] for this typical simplified Tokamak structure. Among them, the Lagrangian approach was found efficient and quite accurate [5]. However, the feasibility of Lagrangian approach for a real Tokamak structure is not clear yet up to now. To calculate the mechanical response of HL-2M VV during PDs, Cai et al. [6] have conducted simulations for HL-2M VV without considering the coupling effect and with an extremely severe disruption case i.e., very large peak plasma current and short decay time, to make the design in the safe side. An additional analysis is necessary with more practical disruption plasma current and considering the coupling effect for the HL-2M Tokamak structure, which is the major aim of this work.

In this paper, the Lagrangian approach adopted for treating the EM-mechanical coupling in VV structural dynamic analysis is first introduced. Then the EM field and structure responses of HL-2M VV during the plasma major disruption (MD) and the vertical displacement disruption (VDE) are computed with and without considering the coupling effect by using an updated numerical code of the Lagrangian approach. In simulation, the plasma movement and current decay during MD and VED are used as the simulation results

* Corresponding Author, chenzm@mail.xjtu.edu.cn 
of the DINA code for design parameters of the HL-2M Tokamak. The maximum dynamic response of displacements and stresses are calculated and the influence of the coupling effect is analyzed finally.

\section{Numerical Approach}

\subsection{Equations of the Lagrangian approach}

In conventional approach, the velocity term $\mathbf{v} \times \mathbf{B}(\mathbf{v}$ is the velocity and $\mathbf{B}$ is the magnetic flux density including both of the confinement field and the induced magnetic field in case of the Tokamak problem), which reflects the effect of the movement and deformation of the conductor, is commonly introduced into the governing equations of EM field to consider the EMmechanical coupling effect,

$$
\begin{aligned}
& \nabla \times\left(\frac{1}{\mu} \nabla \times \mathbf{A}\right)+\gamma\left(\frac{\partial \mathbf{A}}{\partial t}+\nabla \phi-\mathbf{v} \times \mathbf{B}\right)=\boldsymbol{J}_{s} \\
& \nabla \cdot \gamma\left(\frac{\partial \mathbf{A}}{\partial t}+\nabla \phi-\mathbf{v} \times \mathbf{B}\right)=0
\end{aligned}
$$

where $\mathbf{A}$ is the vector potential, $\phi$ is the scalar potential, $\mu, \quad \gamma$ are the magnetic permeability and electric conductivity respectively, and $\boldsymbol{J}_{s}$ is the source electric current such as plasma current.

Eqs. (1), (2) are described under the Eulerian description whose coordinate system is fixed with the earth. The application of the Eulerian description makes the explicit appearance of the velocity term, which not only imposes a numerical burden on equation discretization, but may also worsen the numerical stability [9].

When the spectator is attached to the considered body and pay attention to individual particles in the body as they move through space, the position and physical properties of the particles are described in terms of the material coordinates, i.e., the Lagrangian description [5]. In this case, the derivative of vector $\mathbf{A}$ in Lagrangian description, i.e. the material derivative of $\mathbf{A}$, is equal to the sum of the local rate of change and the convective rate of change of $\mathbf{A}$, as a consequence of the chain rule,

$$
\frac{\mathrm{d} \mathbf{A}}{\mathrm{dt}}=\frac{\partial \mathbf{A}}{\partial \mathrm{t}}+(\mathbf{v} \cdot \nabla) \mathbf{A}
$$

As the velocity term $\mathbf{v} \times \mathbf{B}$ can be expressed as

$$
\mathbf{v} \times \mathbf{B}=\nabla(\mathbf{v} \cdot \mathbf{A})-(\mathbf{v} \cdot \nabla) \mathbf{A}
$$

Eqs. (1) and (2) can be rewritten as follows if we define $\phi=\varphi-\mathbf{v} \cdot \mathbf{A}$

$$
\begin{aligned}
& \nabla \times\left(\frac{1}{\mu} \nabla \times \mathbf{A}\right)+\gamma\left(\frac{\mathrm{d} \mathbf{A}}{\mathrm{dt}}+\nabla \varphi\right)=\mathbf{J}_{\mathrm{s}} \\
& \nabla \cdot \gamma\left(\frac{\mathrm{d} \mathbf{A}}{\mathrm{dt}}+\nabla \varphi\right)=0
\end{aligned}
$$

Above are the governing equations of eddy current problem using the Lagrangian description considering the EM-mechanical coupling effect. One can find that these equations are just in the same form with the EM equations using the Eulerian description but without the velocity term. Therefore, we can realize the Lagrangian approach on the basis of numerical schemes of the conventional Eulerian description simply.

\subsection{Numerical simulation scheme}

The sequential coupling method is usually adopted in the multiple-field coupling problem. According to the sequential coupling method, numerical models with different finite element (FE) types, material constants etc. are established for treating different fields respectively and the results got from one field are transferred to another as input (e.g., loads). In conventional EMmechanical coupling analysis, the EM force obtained from the calculation of EM field is transferred into the mechanical response calculation as loads and in turn the motional eddy currents induced due to the mechanical velocity is applied to EM field as source current. However, the sequential coupling method has some shortcomings for the Eulerian description as pointed out in section 2.1. Therefore, the Lagrangian approach is adopted in this work in the sequential coupling scheme.

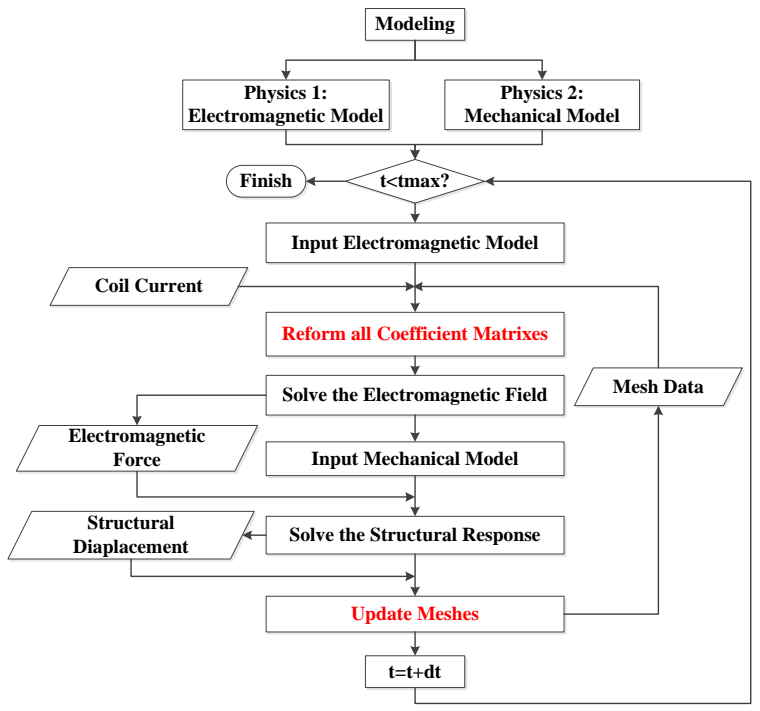

Fig. 1. Flowchart of the EM-mechanical coupling code.

To carry out numerical simulation with Lagrangian approach, the FE meshes of EM field have to be updated according to the deformed configuration of the structure, i.e., to apply moving coordinate system. In practice, the FEM coefficient matrices have to be regenerated at each time step for solving the transient dynamic problem [5]. This can be realized by using the following scheme:

Step 1: Establish an initial EM FEM model and a mechanical FEM model respectively;

Step 2: Input the EM model and update meshes according to the mechanical response of structure at last time step;

Step 3: Regenerate FEM coefficient matrices for the EM field simulation;

Step 4: Solve EM field and calculate EM force; 
Step 5: Input the mechanical model and apply the EM force just obtained at step 4;

Step 6: Solve the structural response and calculate the position and velocity of each structural node;

Step 7: Go to next time point. If the time instant is in the given time range, go back step 2, otherwise, stop and output the results.

Above are the calculating steps with consideration of the coupling effect, while it can also be applied to problem without coupling by neglecting the step 2 and the step 5. In this way, the influence of coupling effect can be investigated by comparing the numerical results with and without the coupling effect considered. The flowchart of this numerical scheme to realize the algorithm listed above is shown in Fig. 1.

Based on the numerical scheme above and the flowchart of Fig.1, a numerical code is developed on the ANSYS platform by using ANSYS parametric design language (APDL). In the code, the Solid 97 and Solid 45 are chosen as the finite element for the EM and the mechanical response simulation respectively and both of them are taken as the 8-node hexahedron elements.

\section{Modeling of HL-2M}

\subsection{Numerical model}

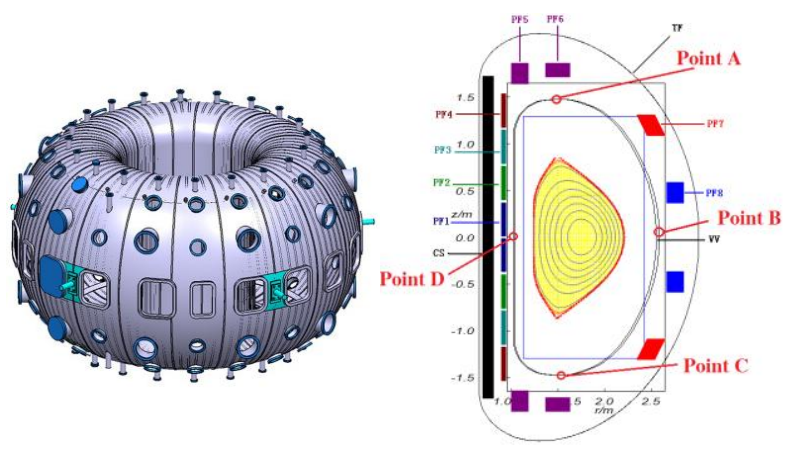

$\begin{array}{ll}\text { (a) } 3 \mathrm{D} \text { view of } \mathrm{VV} & \text { (b) Coils and monitoring points }\end{array}$

Fig. 2. The VV of HL-2M and locations of coils [1].

As shown in Fig. 2(a), the VV of HL-2M is a Dshaped, double thin-wall structure consisting of inner shell, outer shell, reinforcing ribs and ports. The donuts shaped VV is $5.22 \mathrm{~m}$ in outer diameter, $3.02 \mathrm{~m}$ in height and the total wall thickness is $20 \mathrm{~mm}$ (the thickness of the inner shell, outer shell and the distance between the two shells are $5 \mathrm{~mm}, 5 \mathrm{~mm}$ and $10 \mathrm{~mm}$ respectively). The VV is uniformly divided into 20 parts in toroidal direction and supported in 5 equally spaced positions as shown in Fig. 2(a). Inconel 625 steel, whose allowable stress is $414 \mathrm{MPa}$, is chosen to be the material of the VV. The material constants of Inconel 625 steel are shown in Table. 1. On the other hand, the numbers and locations of poloidal field (PF) coils, toroidal field (TF) coils and central field (CS) coil are given in Fig. 2(b).

In view of HL-2M structures, a numerical model consisting of the VV, 16 PF coils, $20 \mathrm{TF}$ coils, CS coil and air region as shown in Fig. 3 is established. To cope with the plasma current during disruption, 42 equivalent circular current filaments distributed uniformly at the central region of the $\mathrm{VV}$ cross-section, and of different amplitudes and time decay are employed and meshed in a similar way with PF coils. The design parameters of coils and currents inside are shown in Table. 2.

Because the VV is supported at five locations equally spaced in toroidal direction in the practical HL-2M and the number of the TF coils is 20, both of the EM and the mechanical field fulfill the $1 / 5$ cyclic symmetry condition. Therefore, a 1/5 model as shown in Fig. 3 is adopted. In this way, the computational burden can be significantly reduced compared with the full model.

Table. 1. The material constants of Inconel 625

\begin{tabular}{ccccc}
\hline $\begin{array}{c}\text { Electric } \\
\text { Conductivity } \\
\gamma / \mathrm{S} \cdot \mathrm{m}^{-1}\end{array}$ & Density & $\begin{array}{c}\text { Young's } \\
\text { Modulus } \\
\text { E/GPa }\end{array}$ & $\begin{array}{c}\text { Poisson } \\
\text { Ratio } \\
v\end{array}$ & $\begin{array}{c}\text { Allowable } \\
\text { Stress } \\
\sigma_{s} / \mathrm{MPa}\end{array}$ \\
\hline $0.78 \times 10^{6}$ & 7860 & 0.189 & 0.3 & 414 \\
\hline
\end{tabular}

Table. 2. The design parameters of coils

\begin{tabular}{ccccc}
\hline Coils & $\mathrm{R}(\mathrm{m})$ & $\mathrm{Z}(\mathrm{m})$ & Turns & Current $(\mathrm{kA})$ \\
\hline PF1 & 0.912 & 0.194 & 28 & -6.667 \\
PF2 & 0.912 & 0.582 & 28 & -5.345 \\
PF3 & 0.912 & 0.970 & 28 & -1.590 \\
PF4 & 0.912 & 1.358 & 28 & -1.130 \\
PF5 & 1.092 & 1.753 & 28 & 11.100 \\
PF6 & 1.501 & 1.790 & 28 & 95.000 \\
PF7 & 2.500 & 1.200 & 26 & -36.000 \\
PF8 & 2.760 & 0.480 & 26 & -24.000 \\
CS & 0.748 & 0 & 96 & 110.000 \\
TF & - & - & $20 * 7$ & 140.000 \\
\hline
\end{tabular}

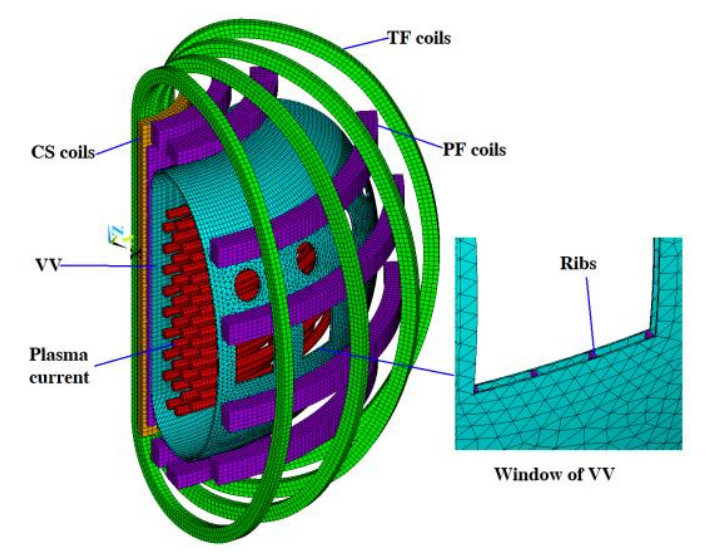

Fig. 3. The 1/5 numerical model of VV of HL-2M.

\subsection{Simulation of plasma currents during disruption}

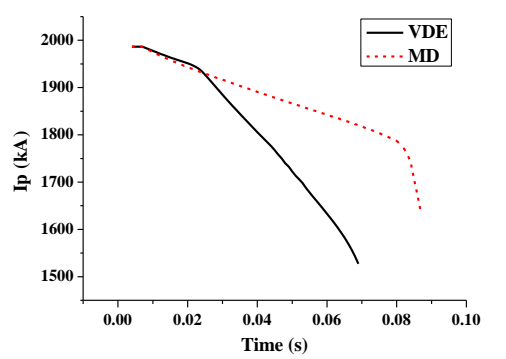

Fig. 4. Total plasma current for VDE and MD. 
The DINA code is widely used to simulate the evolution of the plasma currents and the plasma locations during the plasma disruption [7-8]. To know the evolution of plasma current of HL-2M, two typical cases of disruptions are simulated by using the DINA code, i.e., the MD and VDE. The obtained total current evolutions of these two cases are shown in Fig 4. The plasma current of about 2 MA decreases rapidly during PDs and the VDE shows faster decay. Based on the DINA simulation results, the distributed plasma currents are discretized to 42 current filaments. Each filament has current of different amplitudes and time decay. The amplitudes of each current filament correspond to the space distribution of the plasma current at each time instant, and the time decays in each current filament represent the time evolution of the plasma current. These current filaments are taken as the excitation source of the eddy currents in VV for simulating PDs.

\subsection{Boundary conditions and validation}

The cyclic boundary condition is considered on both sides of the 1/5 (72 degree) model. All degree of DOFs of each pair of nodes on the 0 degree side and the 72 degree side are coupled by the "CPCYC" command of ANSYS. For DOFs coupling, the meshes on the both sides are made exactly the same. In addition, infinite elements are used in the outer layer of the model to simulate the infinite EM boundary. At the nodes laying on the main axis of the model (central axis of CS coil), all DOFs were set fulfill flux parallel condition.

For mechanical response simulation, the same cyclic boundary condition is applied to the both sides of the $1 / 5$ structural model. In addition, the nodes located in a small region at the middle outer surface of the VV (about $0.32 \times 0.5 \mathrm{~m}^{2}$ ), which correspond to one of the five supporting rods, are restrained in both poloidal and toroidal directions to simulate the VV support.

To check the validity of the boundary conditions, the EM field results are compared with those of the full model (360 degree). The relative errors of eddy currents and magnetic flux density are less than $1 \%$. In addition, an approximated analytical solution of eddy currents in a donut shaped tube wall due to a sinusoidal current filament at the toroidal tube axis is derived and is compared with the numerical results using the $1 / 5$ model with the cyclic boundary condition. Even for such an approximated analytical solution, the relative error is less than $5 \%$. This result proved the validity of the boundary condition again.

\section{Numerical Results}

By using the numerical model shown in section 3 and the plasma currents obtained by DINA, the eddy currents, displacement dynamic responses and corresponding stresses are calculated for the HL-2M configuration. To show the results, the displacements and stresses of 4 selected pints, as A, B, C, D (top, right, bottom and left of the outer shell of VV) shown in Fig. 2(b), are extracted. The calculation results during a MD and a
VDE with and without considering the EM-mechanical coupling effect are given and compared as follows.

\subsection{MD}

Fig. 5 shows the time evolution of the displacements at the 4 selected points for cases with and without considering the coupling effect. One can find that the displacements increase exponentially and the maximum displacement appear at the time instant $84 \mathrm{~ms}$. Fig. 6 gives the results of the displacement distribution at time instant $84 \mathrm{~ms}$. It can be found that the displacement at point $\mathrm{D}$ is maximum and that at the point $\mathrm{B}$ is minimum because the region nearby the point $\mathrm{B}$ is constrained. This means that the inner shell of the $\mathrm{VV}$ deforms more significantly. From Fig. 6, the maximum displacement is about $0.16 \mathrm{~mm}$.

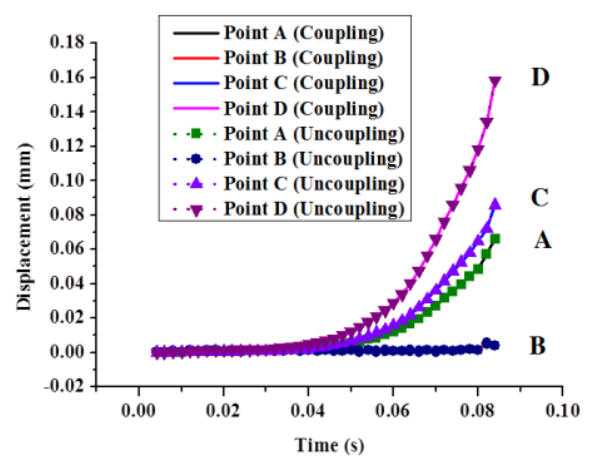

Fig. 5. Displacements of the four points for MD.

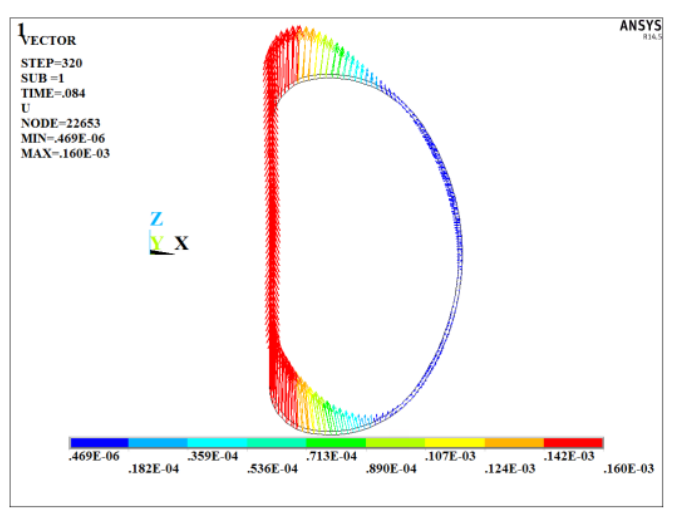

Fig. 6. Displacement distribution for MD (84ms).

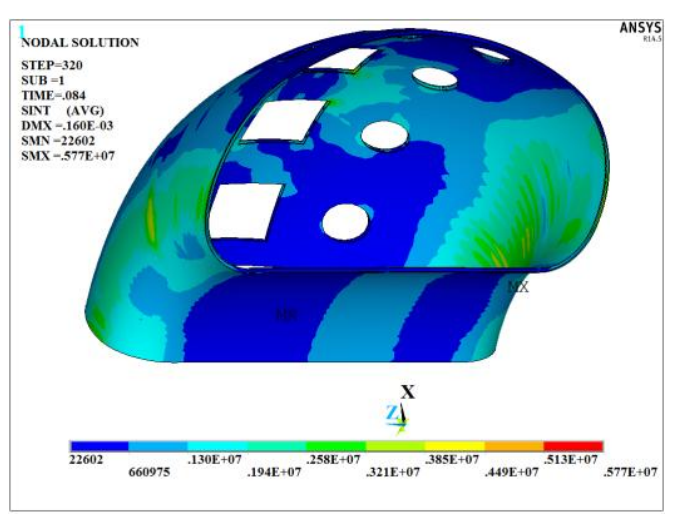

Fig. 7. Stress distribution for MD (84 ms).

Fig. 7 shows the contour map of the stress at time instant $84 \mathrm{~ms}$. From these numerical results, it can be found that the stresses at the point $\mathrm{A}, \mathrm{B}$ and $\mathrm{C}$ are relatively strong compared with point $\mathrm{D}$. In this case, the 
maximum stress is about $5.76 \mathrm{MPa}$ that is far less than the allowable stress of Inconel 625 steel. This result is much smaller than that given in paper [6]. The major reason of this difference is from the very different disruption plasma currents. The plasma current in paper [6] has a much faster decay speed, as only worst case is considered for design purpose.

On the other hand, the numerical results are almost the same for both cases with and without considering the EM-mechanical coupling effect during the plasma MD (Fig.5). This means that the coupling effect does not make a significant influence in this case. The major reason is that the vibration velocity is very small which means a very small motional electric field. In fact, the induced electric field intensity due to the plasma current is in order of $10 \mathrm{~V} / \mathrm{m}$ for this problem while the additional motional electric intensity is in order of $10^{-3}$ $\mathrm{V} / \mathrm{m}$, because the velocity is of order $10^{-2} \mathrm{~m} / \mathrm{s}$ and the poloidal magnetic flux intensity is of order $10^{-1} \mathrm{~T}$. The additional motional electric intensity is too small to give significant influence on the dynamic response in the case.

\subsection{VDE}

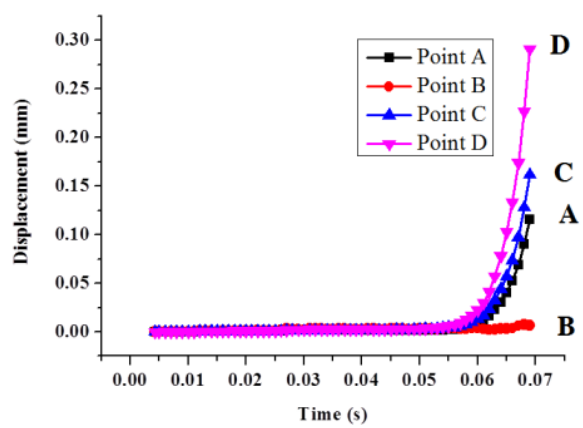

Fig. 8. Displacements of the four points for VDE.

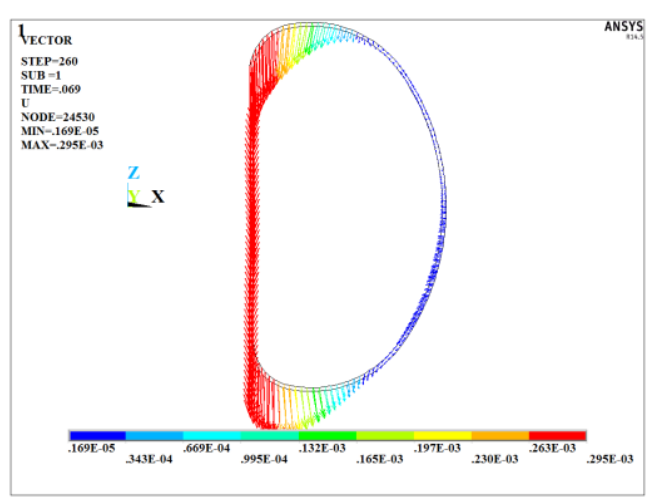

Fig. 9. Displacement distribution for VDE (69 ms).

The displacement responses for VDE case are shown in Fig. 8. Similar with the MD case, the displacement of point $\mathrm{D}$ is maximum and the stresses of point $\mathrm{A}, \mathrm{B}$ and $\mathrm{C}$ are quite big compared with that of point D. Fig. 8 shows that the maximum displacement appears at $69 \mathrm{~ms}$ during the plasma VDE. In addition, the displacements and stresses increase rapidly after $59 \mathrm{~ms}$ due to the rapid change of the global plasma location. In addition, the whole VV moves downward in the case of VDE. From Fig. 9 and the results of stress, the maximum displacement is about $0.3 \mathrm{~mm}$ and the maximum stress is about 11.0 MPa, which are bigger than those of the MD case but still far smaller than the allowable value.

\section{Conclusions}

In this work, the EM field and structural dynamic responses of HL-2M VV during plasma disruptions are simulated by using the Lagrangian approach. The dynamic responses of the displacement and stress of the HL-2M VV are calculated for both cases with and without considering the EM-Mechanical coupling effect. The numerical results show that stresses under the disruptions (MD and VDE) are not significant for HL$2 \mathrm{M} \mathrm{VV}$. In addition, it is also found that the EMmechanical coupling effect does not significantly affect the dynamic response for the HL-2M problem as the vibration velocity is very small.

\section{Acknowledgments}

The authors would like to thank the National Magnetic Confinement Fusion Program of China (2013GB113005), National Science Foundation of China (No. 51277139, 51577139, 11321062 and 51407132) for funding this study.

\section{References}

[1] Liu, D. Q., Ran, H., et al. (2013). Engineering design for the HL-2M tokamak components. Fusion Engineering and Design, 88(6-8), 679-682.

[2] Liu, D. Q., et al. (2015). Assembly study for HL-2M tokamak. Fusion Engineering and Design, 96-97, 298-301.

[3] Li, Q. (2015). The component development status of hl$2 \mathrm{~m}$ tokamak. Fusion Engineering and Design, 96-97, 338-342.

[4] Takagi, T. (1982). Summary of the results for magnetic damping in torsional mode (team problem 16). Compel the International Journal for Computation and Mathematics in Electrical and Electronic Engineering, 14(2-3), 77-89.

[5] Li, W., Yuan, Z. and Chen, Z. (2014). A moving coordinate numerical approach for analyses of electromagnetomechanical coupled behavior of structures in a strong magnetic field aiming at application to tokamak structures. Plasma Science and Technology, 16(12), 1163-1170.

[6] Cai, L., et al. (2013). Preliminary Calculation of Electromagnetic Loads on Vacuum Vessel of HL-2M. Plasma Science and Technology 15(3), 271-276.

[7] Mitrishkin, Y. V., Korostelev, A. Y., Dokuka, V. N. and Khayrutdinov, R. R. (2010). Design and modeling of ITER plasma magnetic control system in plasma current ramp-up phase on DINA code. Proceedings of the IEEE Conference on Decision and Control ,1354-1359.

[8] V.E. Lukash, R.R. Khayrutdinov. (2007). Model of real time ITER plasma position, shape and current control on base of DINA code. The 34th EPS Conference on Plasma Phys. Vol. 31F, P-5.148.

[9] Tanaka, Y., Horie, T., Niho, T., Shintaku, E. and Fujimoto, Y. (2004). Stability of augmented staggered approach for electromagnetic and structural coupled problem. IEEE Transactions on Magnetics, 40(2), 549-552. 